\title{
Improvement in the intense pulsed emission stability of grown CNT films via an electroless plated Ni layer
}

\author{
ZENG FanGuang $^{1 *}$, LI Xin $^{2}$, LIU WeiHua ${ }^{2}$, QIAO ShuZhen ${ }^{1}$, MA HuaLi ${ }^{1}$, ZHANG Rui $^{1}$, \\ XIA LianSheng ${ }^{3}$, CHEN Yi $^{3}$, LIU XingGuang ${ }^{3}$ \& ZHANG Huang ${ }^{3}$ \\ ${ }^{1}$ Department of Mathematics and Physics, Zhengzhou Institute of Aeronautical Industry Management, Zhengzhou 450015, China; \\ ${ }^{2}$ School of Electronic and Information Engineering, Xi'an Jiaotong University, Xi'an 710049, China; \\ ${ }^{3}$ Institute of Fluid Physics, Chinese Academy of Engineering Physics, Mianyang 621900, China
}

Received February 18, 2011; accepted April 15, 2011

Carbon nanotube (CNT) films were grown on silicon wafers with and without a nickel layer (Si-CNT and Ni-CNT) via the pyrolysis of iron phthalocyanine. The nickel layer was prepared using the electroless plating method. To study the emission stability of Si-CNT and Ni-CNT cathodes during intense pulsed emission, emission characteristics were measured repeatedly with a diode structure using a Marx generator as a voltage source. For the peak values of the pulsed voltage, which were in the range between 1.62-1.66 MV (corresponding to electric field intensities between 11.57-11.85 V/ $\mu \mathrm{m}$ ), the first cycle emission current was 109.4 A for Si-CNT and 180.5 A for Ni-CNT. By comparing the normalized emission currents of the Si-CNT and Ni-CNT cathodes, the improvement in the emission stability can be easily quantified. The number of emission cycles necessary for the peak current to decay from $100 \%$ to $50 \%$ increased from $\sim 3$ for Si-CNT to $\sim 11$ for a Ni-CNT film.

carbon nanotube, nickel layer, intense pulsed emission, improved emission stability, normalized current

Citation: Zeng F G, Li X, Liu W H, et al. Improvement in the intense pulsed emission stability of grown CNT films via an electroless plated Ni layer. Chinese Sci Bull, 2011, 56: 2379-2382, doi: 10.1007/s11434-011-4567-z

Carbon nanotubes (CNTs) have attracted a great deal of attention because of their potential use in emission applications. This is because of their nanometer size scale, high aspect ratio, high mechanical strength, good conductance and high chemical stability [1-3]. These characteristics give CNTs application in many devices, such as field emission displays, high-resolution electron-beam instruments, lamps, $\mathrm{X}$-ray sources, and microwave amplifier tubes [4-9]. Emission stability is a parameter that is vital to electron emission applications, especially in intense pulsed emission systems. Unfortunately, there is currently no reported research on intense pulsed emission stability.

In this paper, we study the emission stability of CNT films grown on silicon wafers during intense pulsed emission, and report on improvements in intense pulsed emis-

*Corresponding author (email: fgzeng@ sina.com) sion stability when a nickel (Ni) layer is used as the growth substrate.

\section{Experimental}

\subsection{Sample preparation}

CNT films were grown on single-sided polished silicon wafers (n-type, (100) oriented, resistivities between $10^{-2}-10^{-3}$ $\Omega \mathrm{cm}^{-1}, 2$-inch diameter) with and without a Ni layer (Si-CNT and Ni-CNT). The Ni layer that was used for CNT film growth was prepared on a $\mathrm{Si}$ wafer using an electroless plating process. The plating solution was composed of nickel sulfate, $\mathrm{NaH}_{2} \mathrm{PO}_{2}$, and trisodium citrate. The $\mathrm{pH}$ value of the solution was adjusted to be between $8-10$ by adding ammonia to the solution. A palladium catalyst was used for the Ni plating. The CNT film was synthesized via the 
pyrolysis of iron phthalocyanine $(\mathrm{FePc})[10]$

\subsection{Morphology of the samples}

A scanning electron microscope (SEM, JEOL JSM-6700F, Tokyo, Japan) was used to study the morphologies of the $\mathrm{Ni}$ layer, Si-CNT and Ni-CNT films.

\subsection{Intense pulsed emission characteristics}

The intense pulsed emission characteristics were measured using a diode structure with a pulse generator under a vacuum of $\sim 5 \times 10^{-4} \mathrm{~Pa}$. The CNT cathodes were used in single pulse mode (i.e., only one voltage pulse was applied for each test procedure) during the intense pulsed emission measurement. To study the emission stability, we repeatedly measured the emission currents of the Si-CNT and Ni-CNT films under voltage supplied by a Marx generator. All the pulsed voltages have the same half-value width, which was $100 \mathrm{~ns}$.

\section{Results and discussion}

\subsection{Morphology of the samples}

Figure 1 shows SEM images of the Ni substrate. It can be seen from Figure 1(a) that Ni the layer is homogeneous and compact. We estimated from Figure 1(b) that the size range of Ni particles was $150-300 \mathrm{~nm}$.

SEM images of the Si-CNT and Ni-CNT films are shown in Figure 2. It can be seen that CNTs in both the Si-CNT and Ni-CNT films are aligned perpendicularly to the substrates and have a height of several microns.

\subsection{Stability characteristics of the intense pulsed emis- sion current}

Although all the measurements of emission current were performed using the same voltages from the Marx generator, the pulsed voltages between anode and cathode were different for each emission cycle. The range of peak values for the pulsed voltage and electric field intensity for all the emission stability measurements were 1.62-1.66 MV and $11.57-11.85 \mathrm{~V} / \mu \mathrm{m}$.

Figure 3 shows the results of repeated emission current measurements for Si-CNT and Ni-CNT cathodes. The absence of a data point for cycle number 3 in Figure 3 (b) is because the maximum current datum was over the upper limit of the recorder. It can be seen in Figures 3(a) and (b) that the peak current values of the first emission cycle of the Si-CNT and Ni-CNT films are 109.4 and 180.5 A. The peak values of the emission current generally decayed with
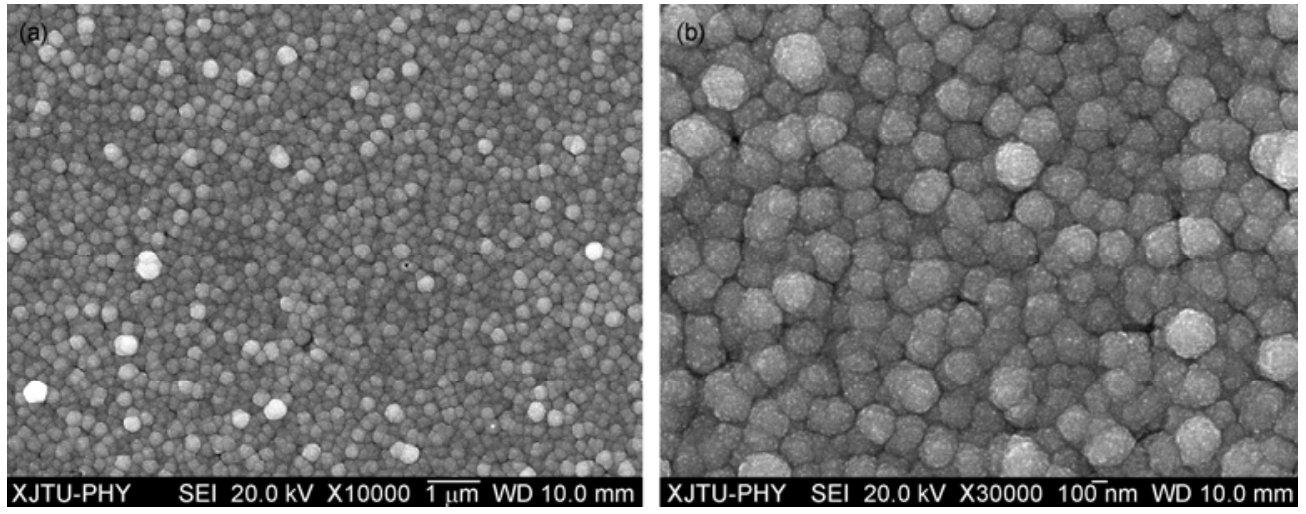

Figure 1 SEM images of the Ni layer. (a) Low- and (b) high-magnification images.
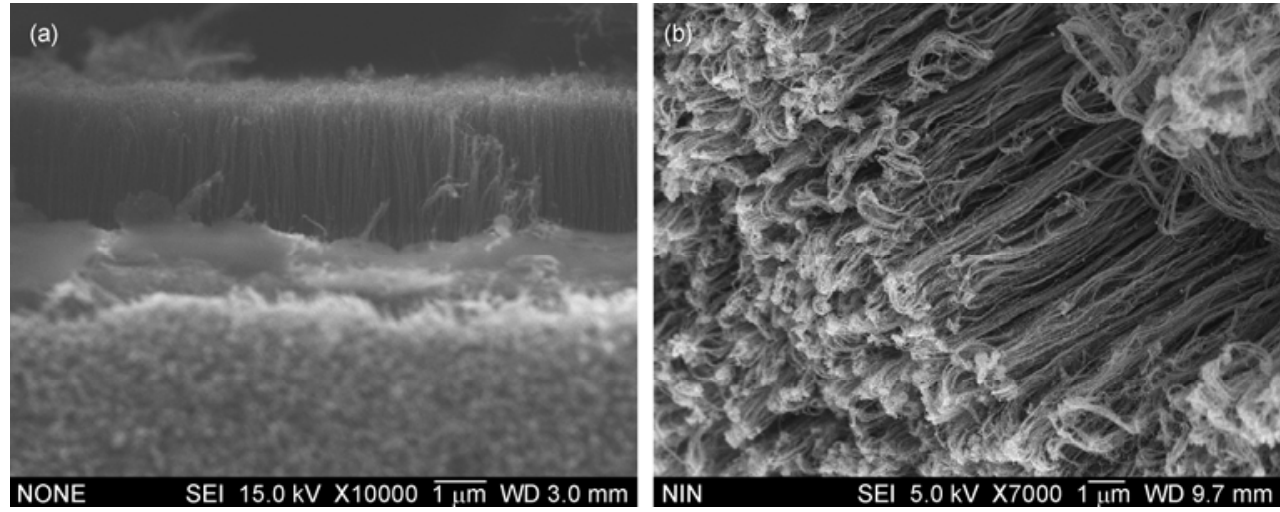

Figure 2 SEM images of the Si-CNT (a) and Ni-CNT (b) films. 

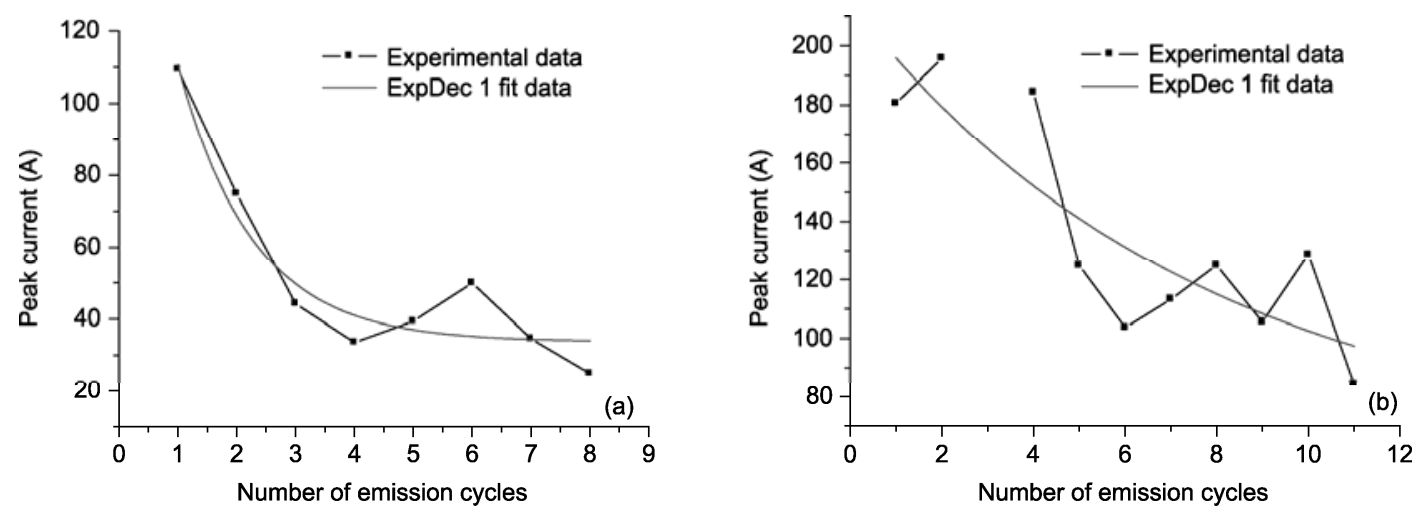

Figure 3 Current stability characteristics for the Si-CNT (a) and Ni-CNT (b) cathodes in an intense pulsed emission mode.

increasing measurement number (emission cycle number). There was some fluctuation in emission current in the measurement results. The fluctuation in the emission current can be attributed to fluctuations in the applied pulsed voltage. This affected the current measurement results, but it did not change the current decay trend.

Because a formula for emission current decay over time in an intense pulsed emission mode could not be found, we described the decay using an exponential decay model, which was used in previous work [11]. Assuming the emission current decayed according to a first order exponential decay model, we can get fitting curves using the first order "Fit Exponential Decay" function (ExpDec 1 model) in Origin 6.1 (Northampton, MA, USA).

The ExpDec 1 model can be described using

$$
y=y_{0}+A_{1} \mathrm{e}^{-x / t_{1}} .
$$

According to eq. (1) and the fitted results, the decay formulas for emission current of the Si-CNT and Ni-CNT cathodes can be written as

$$
\begin{aligned}
I_{1} & =I_{10}+A_{11} \mathrm{e}^{-x / t_{1}} \\
& =33.75509+169.06883 \mathrm{e}^{-x / 1.27035}(\mathrm{~A}), \\
I_{2} & =I_{20}+A_{21} \mathrm{e}^{-x / t_{2}} \\
& =60.69897+153.88518 \mathrm{e}^{-x / 7.69049}(\mathrm{~A}),
\end{aligned}
$$

where $I_{1}$ is the peak current of the Si-CNT cathode; $I_{2}$ is the peak current of the Ni-CNT cathode; and $x$ is the emission cycle number corresponding to $I_{1}$ and $I_{2}$.

The smooth curves labeled "ExpDec 1 fit Data" in Figure 3(a) and (b) are the first order exponential decay model fits for the Si-CNT and Ni-CNT cathodes. We found from the experimental and fitted curves that the decay speed of the Ni-CNT film is lower than that of the Si-CNT film.

\subsection{Comparison of stability characteristics}

To compare the levels of emission current for the samples, we used the normalized emission current. The normalized emission current is defined as the ratio of the current values of each point in fit curve to that of the first point. The normalized current is actually a dimensionless relative current, given by

$$
I_{n}=\frac{I}{I_{\mathrm{Max}}},
$$

where $I_{n}$ is the normalized current; $I$ is the fitted current; and $I_{\mathrm{Max}}$ is the initial value (maximum value) for the fitted current.

To compare the decay trends directly, the normalized currents of Si-CNT and Ni-CNT cathodes were plotted together versus emission cycle number. The comparison curves are shown in Figure 4.

Because the normalized emission current denotes the percentage of the current value relative to the initial value, the emission cycle numbers corresponding to the same normalized current can be used for a comparison of the decay speed. Using $50 \%$ as a criterion, the emission cycle numbers that corresponded to a $50 \%$ decrease are $\sim 3$ for Si-CNT and $\sim 11$ for Ni-CNT.

\subsection{Analysis of the mechanism for emission stability improvement}

Generally, the factors that affect the conventional field

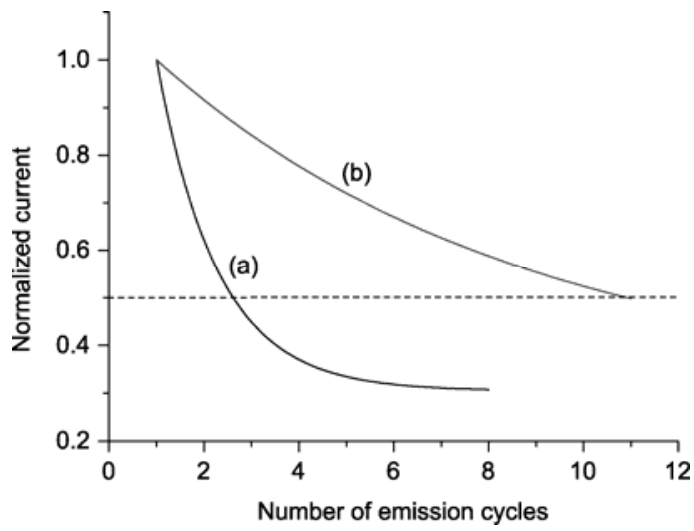

Figure 4 Comparison of the emission current decay trends for Si-CNT (a) and $\mathrm{Ni}-\mathrm{CNT}$ (b). 
emission stability of DC modes include surface reactions between CNTs and gas molecules [12] and damage to the nanotubes [13] or the CNT-substrate interface [14]. Often, such damage can be caused by resistive heating from high-current field emission. The passivation of the CNT surface with amorphous carbon [15] can help shield the CNTs from reacting with ambient gas, and thus results in more stable field-emission behavior. However, the adhesion of CNTs to substrate is also critical for stability [16]. The strong electrostatic force exerted on the CNTs during emission can separate the CNTs from the substrate, causing current decay and arcing.

In intense pulsed emission modes, the CNT cathode emits a very high current. The damage caused resistive heating may be the primary factor in the decay of the emission current. Introducing a Ni layer has two positive effects resulting in improvement to the emission stability: (1) The resistance of the Ni layer is lower than that of the Si substrate. This can decrease the Joule heating during electron emission. (2) According to our experimental results, the bonding of CNTs to the Ni layer is better than that to the $\mathrm{Si}$ substrate. This can decrease the resistance of CNT-substrate interface. These two effects decrease the damage caused by resistive heating from the high-current field emission and improve the emission stability.

Moreover, when the density of the CNTs increases, the field emission intensity for the CNT cathode will be reduced through the field screening effect $[17,18]$. This results in a higher turn-on voltage and lower emission current. For extremely dense CNT films, only the few CNTs which extend out past the surface the film have strong emission capabilities. The probability these CNTs being damaged will be higher because they suffer higher emission currents, especially in an intense pulsed emission mode. Therefore, if the growth density of the CNTs can be restricted to the proper value, the emission characteristics and the emission stability can be further improved.

\section{Conclusion}

In summary, a Ni layer can be deposited on the surface of silicon wafer using the electroless plating method. CNT films can grow directly on silicon wafer with or without a Ni layer via the pyrolysis of FePc. The emission stability of CNT films grown on $\mathrm{Si}$ wafers during intense pulsed emission can be improved by introducing a $\mathrm{Ni}$ layer prior to CNT growth. The normalized emission current was used to contrast the differences in the decay trends in emission current of Si-CNT and Ni-CNT cathodes. The number of emis- sion cycles corresponding to a normalized current decay from $100 \%$ to $50 \%$ increased from $\sim 3$ for a Si-CNT film to $\sim 11$ for a Ni-CNT film.

This work was supported by the National Natural Science Foundation of China (51072184, 50972132, 51002143 and 60801022), the Aeronautical Science Foundation of China (2009ZE55003 and 2010ZF55013) and the Basic and Advanced Technology Program of Henan (092300410139). The authors would like to thank Pan Haifeng from the Institute of Fluid Physics, Chinese Academy of Engineering Physics for help in the measurement of the intense pulsed emission characteristics.

1 Iijima S. Helical microtubes of graphitic carbon. Nature, 1991, 354: 56-58

2 De Heer W A, Chatelain A, Ugarte D. A carbon nanotube field emission electron source. Science, 1995, 270: 1179-1180

3 Bonard J M, Croci M, Klinke C, et al. Carbon nanotube films as electron field emitters. Carbon, 2002, 40: 1715-1728

4 de Jonge N, Bonard J M. Carbon nanotube electron sources and applications. Philos Trans R Soc London A, 2004, 362: 2239-2266

5 de Jonge N, Lamy Y, Schoots K, et al. High brightness electron beam from a multi-walled carbon nanotube. Nature, 2002, 420: 393-395

6 Teo K B K, Chhowalla M, Amaratunga G A J, et al. Fabrication and electrical characteristics of carbon nanotube-based microcathodes for use in a parallel electron-beam lithography system. J Vac Sci Technol B, 2003, 21: 693-697

7 Cheng Y, Zhang J, Lee Y Z, et al. Dynamic radiography using a carbonnanotube-based field-emission x-ray source. Rev Sci Instrum, 2004, 75: 3264-3267

8 Choi W B, Chung D S, Kang J H, et al. Fully sealed, high-brightness carbon-nanotube field-emission display. Appl Phys Lett, 1999, 75: 3129-3131

9 Teo K B K, Minoux E, Hudanski L, et al. Microwave devices: Carbon nanotubes as cold cathodes. Nature, 2005, 437: 968

10 Li D C, Dai L M, Huang S M, et al. Structure and growth of aligned carbon nanotube films by pyrolysis. Chem Phys Lett, 2000, 316: 349-355

11 Weon B M, Je J H. Time-dependent current-voltage relation in electron guns. J Appl Phys, 2005, 97: 036101

12 Dong C, Gupta M C. Influences of the surface reactions on the field emission from multiwall carbon nano-tubes. Appl Phys Lett, 2003, 83: $159-161$

13 Nilsson L, Groening O, Groening P, et al. Collective emission degradation behavior of carbon nanotube thin-film electron emitters. Appl Phys Lett, 2001, 79: 1036-1038

14 Rao A M, Jacques D, Haddon R C, et al. In situ-grown carbon nanotube array with excellent field emission characteristics. Appl Phys Lett, 2000, 76: 3813-3815

15 Jo S H, Tu Y, Huang Z P, et al. Correlation of field emission and surface microstructure of vertically aligned carbon nanotubes. Appl Phys Lett, 2004, 84: 413-415

16 Cheng Y, Zhou O. Electron field emission from carbon nanotubes. CR Phys, 2003, 4: 1021-1033

17 Choi J H, Choi S H, Han J H, et al. Enhanced electron emission from carbon nanotubes through density control using in situ plasma treatment of catalyst metal. J Appl Phys, 2003, 94: 487-490

18 Kong B Y, Seon J Y, Lee S H, et al. Density control of highly populated carbon nanotubes grown by thermal chemical vapor deposition to improve their field emission characteristics. J Korean Phys Soc, 2004, 45: 1580-1583

Open Access This article is distributed under the terms of the Creative Commons Attribution License which permits any use, distribution, and reproduction in any medium, provided the original author(s) and source are credited. 